\title{
Character difference digraphs over finite fields
}

\author{
Risto Atanasov $(*)$ - Mark Budden (**) - Joshua Lambert $(* * *)$
}

ABstract - We generalize the notion of Paley digraphs by defining and studying character difference digraphs for characters defined on the multiplicative groups of finite fields.

Mathematics Subject Classification (2010). 05C30, 05C20, 11T24; 05C25, 11R16.

KeYwords. Paley graphs, Jacobi sums.

\section{Introduction}

The study of digraphs provides a proving ground where mathematicians' ability to bind together multiple disciplines of mathematics becomes evident. Early ties introduced themselves when Graham and Spencer [5] brought forth the idea of using quadratic residues to construct a tournament with $p$ vertices where $p \equiv 3(\bmod 4)$ is a prime. These tournaments were appropriately named Paley digraphs in honor of the late Raymond Paley, who used quadratic residues 38 years earlier to construct Hadamard matrices [11]. Prior to the introduction of Paley digraphs, there was much interest in the class of undirected graphs known as Paley graphs [12, 4], given their ability to draw together number theory and graph theory. Lim

(*) Indirizzo dell'A.: Department of Mathematics and Computer Science, Western Carolina University, Cullowhee, NC 28723, USA.

E-mail: ratanasov@email.wcu.edu

(**) Department of Mathematics and Computer Science, Western Carolina University, Cullowhee, NC 28723, USA.

E-mail: mrbudden@email.wcu.edu

(***) Department of Mathematics, Armstrong Atlantic State University, 11935 Abercorn St., Savannah, GA 31419, USA.

E-mail: Joshua.Lambert@armstrong.edu 
and Praeger [9] recently generalized the notion of these Paley graphs and appropriately named this class of graphs generalised Paley graphs.

Similar to their predecessors, Lim and Praeger began with graphs having a vertex set given by $\mathbb{F}_{q}$, where $q=p^{f}$ for an odd prime $p$. Rather than using quadratic residues to create edges in their corresponding graph, they formed an edge between vertices $a$ and $b$ if and only if their difference is in a fixed subgroup $S$ of $\mathrm{F}_{q}^{\times}$of even order. Our approach will differ from that of Lim and Praeger as we shall consider a character (group homomorphism) $\chi: \mathbb{F}_{q}^{\times} \longrightarrow \mathbb{C}^{\times}$and identify the subgroup $S$ with the kernel $\operatorname{Ker}(\chi)$ to create a class of digraphs called character difference digraphs.

The necessity of $\operatorname{Ker}(\chi)$ having even order in Lim and Praeger's generalised Paley graphs stem from $-1 \in \operatorname{Ker}(\chi)$ (as can be confirmed by noting that $\mathrm{F}_{q}^{\times}$is cyclic). We shall further generalize the graphs in question by removing the assumption of even order, which explains our focus on digraphs since only one of $a-b$ and $b-a$ may now be in the kernel. The character difference digraphs $D_{\chi}$ we consider have vertex set $\mathbb{F}_{q}$ and an arc from $a$ to $b$ exists if and only if $b-a \in \operatorname{Ker}(\chi)$. After a brief description of some of the basic properties afforded by such digraphs, our attention will turn to the enumeration of the directed triangles (3-cycles). Similar to the experience of Graham and Spencer, we shall call upon an interesting number-theoretic problem to determine the number of directed triangles. Alongside these calculations we determine the exact number of such directed triangles for quadratic, cubic, and quartic characters on $\mathbb{F}_{q}^{\times}$. Our calculations shall then utilize the work of Davenport and Hasse [3] to relate the number of directed triangles in such digraphs over $\mathbb{F}_{q}$ to that of the corresponding digraphs over $\mathbb{F}_{r}$, where $r=q^{s}$.

\section{Background and Notations}

Throughout the remainder of this paper, we assume that $p$ is an odd prime, $q=p^{f}$, and $r=q^{s}$. Suppose that $\chi: \mathbb{F}_{q}^{\times} \longrightarrow \mathbb{C}^{\times}$is a character of order $r_{\chi}>1$ when viewed as an element of the character group for $\mathbb{F}_{q}^{\times}$. We see that $r_{\chi}$ divides $q-1$ and the image of $\chi$ lies in the set

$$
\mu_{r_{\chi}}:=\left\{\zeta_{r_{\chi}}^{j} \mid j=0,1, \ldots, r_{\chi}-1\right\}
$$

of $r_{\chi}$-th roots of unity in $\mathbb{C}^{\times}$. As such, it follows that the inverse character of $\chi$ in the character group of $\mathbb{F}_{q}^{\times}$is equal to the conjugate character: $\chi^{-1}=\bar{\chi}$. 
The digraphs $D_{\chi}$ that will be the focus of this article have vertex set $V\left(D_{\chi}\right):=\mathrm{F}_{q}$ and ares given by

$$
E\left(D_{\chi}\right):=\{(a, b) \mid b-a \in \operatorname{Ker}(\chi)\} .
$$

Since the kernel has order $|\operatorname{Ker}(\chi)|=\frac{q-1}{r_{\chi}}$ and $D_{\chi}$ is regular, we observe that the number of arcs is given by $\left|E\left(D_{\chi}\right)\right|=\frac{q(q-1)}{r_{\chi}}$.

While recognizing $b-a=-(a-b)$, we notice that the determination of whether or not $-1 \in \operatorname{Ker}(\chi)$ provides us with two fundamentally different classes of digraphs. When $\chi(-1)=1$, we see that the corresponding character difference digraph is symmetric. Meanwhile, the case where $\chi(-1)=-1$ provides us with at most one of $(a, b)$ and $(b, a)$ being in $E\left(D_{\chi}\right)$.

A familiarity with this class of digraphs immediately becomes prevalent when considering the special case when $\chi$ maps $\mathbb{F}_{p}^{\times}$to $\mathbb{C}^{\times}$. We note that the associated digraphs are circulant and since $p$ is a prime, we know that $D_{\chi}$ is a $\frac{p-1}{r_{\chi}}$-strong hamiltonian digraph. In fact, for each $n \in \operatorname{Ker}(\chi)$, the additive order of $n$ in $\mathbb{F}_{p}$ leads to a hamiltonian cycle on the vertices

$$
0, n, 2 n, \ldots,(p-1) n .
$$

Furthermore, since such an $n$ defines a unique generator for a cycle, no two distinct elements in $\operatorname{Ker}(\chi)$ will give the same hamiltonian cycle. Thus, $D_{\chi}$ decomposes into $\frac{p-1}{r_{\chi}}$ hamiltonian cycles.

Even the general form of our character having domain $\mathbb{F}_{q}^{\times}$has similar characteristics to its circulant counterpart. In the process of proving other properties of $D_{\chi}$, it will be helpful for us to start with the digraph on $\mathbb{F}_{p}$, then lift the character to form the corresponding digraph on $\mathbb{F}_{q}$. Since character sums will play an important role in our computations, we shall refer to the work of Davenport and Hasse [3], which described the relationship between the Jacobi sums over finite fields and that of their finite extensions. In order to describe their results, note that the field extension $\mathbb{F}_{r} / \mathbb{F}_{q}$ is cyclic with $\alpha \mapsto \alpha^{q}$ serving as a generator for $\operatorname{Gal}\left(\mathbb{F}_{r} / \mathbb{F}_{q}\right)$. We can use the norm map

$$
N_{\mathrm{F}_{r} / \mathbb{F}_{q}}(\alpha):=\alpha \cdot \alpha^{q} \cdots \alpha^{q^{s-1}}
$$

to develop an understanding of the connection between the multiplicative structures of $\mathrm{F}_{r}$ and $\mathbb{F}_{q}$. Throughout this article, we shall take advantage of the shorthand notation $N_{r / q}$ to denote $N_{\mathbb{F}_{r} / \mathbb{F}_{q}}$. It is worth noting that for all 
$\alpha, \beta \in \mathbb{F}_{r}$, we have

$$
N_{r / q}(\alpha \beta)=N_{r / q}(\alpha) N_{r / q}(\beta),
$$

and the homomorphism $N_{r / q}: \mathbb{F}_{r}^{\times} \longrightarrow \mathbb{F}_{q}^{\times}$is surjective.

Given a nontrivial character $\chi: \mathbb{F}_{q}^{\times} \longrightarrow \mathbb{C}^{\times}$of order $r_{\chi}>1$, we may lift $\chi$ to a character $\psi: \mathbb{F}_{r}^{\times} \longrightarrow \mathbb{C}^{\times}$by defining $\psi:=\chi \circ N_{r / q}$. Since $N_{r / q}$ is surjective, it follows that $r_{\chi}=r_{\psi}$. Davenport and Hasse [3] (also, see Section 4.6 of [8]) explained how one lifts the corresponding Jacobi sums for the finite fields and characters in question. Recall that if $\chi$ and $\chi^{\prime}$ are two nontrivial characters on $\mathbb{F}_{q}^{\times}$, the Jacobi sum of $\chi$ and $\chi^{\prime}$ is defined to be

$$
J\left(\chi, \chi^{\prime}\right):=-\sum_{\substack{n \in \mathbb{F}_{q}^{\times} \\ n \neq 1}} \chi(n) \chi^{\prime}(1-n) .
$$

At first, the minus sign in this definition seems out-of-place, but its existence greatly simplifies the statement of the results of Davenport and Hasse [3]. Namely, we have

$$
J\left(\psi, \psi^{\prime}\right)=J\left(\chi, \chi^{\prime}\right)^{s}
$$

where $\psi=\chi \circ N_{r / q}$ and $\psi^{\prime}=\chi^{\prime} \circ N_{r / q}$ are the lifted characters of $\chi$ and $\chi^{\prime}$ to $\mathbb{F}_{r}^{\times}$.

\section{Enumeration of Directed Triangles}

The art of counting triangles can be traced back to the early study of digraphs. In 1940, Kendall and Smith [7] brought an interesting twist to this task by demonstrating that any n-vertex tournament $T$ has a total of

$$
\left(\begin{array}{l}
n \\
3
\end{array}\right)-\sum_{v \in V(T)}\left(\begin{array}{c}
d^{+}(v) \\
2
\end{array}\right)
$$

directed triangles, where $d^{+}(v)$ denotes the out-degree of $v$. These techniques have been improved upon throughout the years on different classes of digraphs, which leads us to our main goal: to provide a formula for the number of directed triangles in character difference digraphs. The following theorem generalizes the approach used by Maheswari and Lavaku in [10], in which they enumerated triangles in certain Paley graphs.

TheOREM 3.1. Let $\chi: \mathbb{F}_{q}^{\times} \longrightarrow \mathrm{C}^{\times}$be a character of order $r_{\chi}$. Then the number of directed triangles in $D_{\chi}$ is given by

$$
T\left(D_{\chi}\right)=\frac{q(q-1)}{3 r_{\chi}} M(\chi)
$$


where $M(\chi)$ denotes the cardinality of the set $\left\{b \in \mathbb{F}_{q}^{\times} \backslash\{1\} \mid \chi(b-1)=\right.$ $\chi(-b)=1\}$.

PRoof. To count the number of directed triangles, we consider first the fundamental directed triangles given by

$$
\Delta_{1}:=\{(0,1, b) \mid b-1,-b \in \operatorname{Ker}(\chi)\} .
$$

The number of such directed triangles is equal to the number of pairs $b-1,-b \in \operatorname{Ker}(\chi)$, which we denote by $M(\chi)$. For any $a \in \operatorname{Ker}(\chi)$, define the set of directed triangles

$$
\Delta_{a}:=\{(0, a, b) \mid b-a,-b \in \operatorname{Ker}(\chi)\} .
$$

It is easily confirmed that the map

$$
(0,1, b) \mapsto(0, a, a b)
$$

defines a bijection $\Delta_{1} \longrightarrow \Delta_{a}$, from which we see that $\Delta_{a}$ also has cardinality $M(\chi)$. Thus, the total number of directed triangles that contain 0 as a vertex is given by

$$
|\operatorname{Ker}(\chi)| \cdot M(\chi)=\frac{q-1}{r_{\chi}} M(\chi) .
$$

Since the graph $D_{\chi}$ is regular, every vertex is contained in the same number of directed triangles. Counting the total number of directed triangles through each vertex and noting that each triangle contains 3 vertices, we obtain the desired result.

Theorem 3.1 further demonstrates the differences in the two fundamental classes of character difference digraphs. Notice that if $-1 \in \operatorname{Ker}(\chi)$ our corresponding directed triangles occur when $b-1, b \in \operatorname{Ker}(\chi)$. In other words, our calculation will be based upon the occurrence of consecutive pairs of elements being in the kernel of our corresponding character. Such calculations can be found for the case of quartic residue graphs in [2]. Although the approach may be similar to the aforementioned calculations, determining the explicit value of $M(\chi)$ can be rather challenging. We shall now try to generalize the approach described in [2], providing a description of $M(\chi)$ that depends upon the evaluation of certain Jacobi sums.

Since $\chi$ takes on its values in $\mu_{r_{\gamma}}$, consider the polynomial

$$
\Psi(x)=x^{r_{x}-1}+x^{r_{x}-2}+\cdots+x+1,
$$

which contains all of the $r_{\chi}^{\text {th }}$ roots of unity as roots, with the exception of 1 . 
Hence, for any $n \in \mathbb{F}_{q}^{\times}$, we have

$$
\Psi(\chi(n))=\left\{\begin{array}{cl}
r_{\chi} & \text { if } n \in \operatorname{Ker}(\chi) \\
0 & \text { if } n \notin \operatorname{Ker}(\chi) .
\end{array}\right.
$$

Thus, we are able to identify $M(\chi)$ with the following summation:

$$
M(\chi)=\frac{1}{r_{\chi}^{2}} \sum_{\substack{n \in \mathbb{F}_{q}^{\times} \\ n \neq 1}} \Psi(\chi(-n)) \Psi(\chi(n-1)) .
$$

Once the product in this sum is expanded, one can focus on the individual sums in the expansion. An essential tool for many of the evaluations is the orthogonality relation

$$
\sum_{n \in \mathbb{F}_{q}^{\times}} \chi(n)=\left\{\begin{array}{cc}
0 & \text { if } \chi \neq \varepsilon \\
q-1 & \text { if } \chi=\varepsilon
\end{array}\right.
$$

where $\varepsilon$ is the trivial character. For more complicated sums, it will often be necessary to appeal to standard manipulations of Jacobi sums.

In order to illustrate how one may obtain a closed-form for $M(\chi)$ using the above summation, we explicitly evaluate $T\left(D_{\chi}\right)$ for some low-order cases. Namely, we focus on the quadratic, cubic, and quartic cases in the following sections.

\section{Quadratic Characters}

Graham and Spencer's results [5] called upon the use of quadratic residues with $p$ vertices where $p$ is a prime with $p \equiv 3(\bmod 4)$. These digraphs fall under the guise of the quadratic character with the vertex set given by $\mathbb{F}_{p}$. However, we can further generalize this set of graphs along with known formulas for the number of triangles in such tournaments with a more concise counterpart.

Let $\chi_{2}: \mathbb{F}_{q}^{\times} \longrightarrow \mathrm{C}^{\times}$be the unique character of order 2 , where we continue to assume $q=p^{f}$. After expanding $M\left(\chi_{2}\right)$, we must compute the four separate sums that arise in

$$
M\left(\chi_{2}\right)=\frac{1}{4} \sum_{\substack{n \in F_{q}^{\times} \\ n \neq 1}}\left(1+\chi_{2}(-n)+\chi_{2}(n-1)+\chi_{2}(-n) \chi_{2}(n-1)\right) .
$$


The first sum is

$$
\sum_{\substack{n \in \mathbb{F}_{q}^{\times} \\ n \neq 1}} 1=q-2
$$

The second and third sums are computed using the orthogonality relation, giving:

$$
\sum_{\substack{n \in F_{q}^{\times} \\ n \neq 1}} \chi_{2}(-n)=\sum_{\substack{n \in \mathbb{F}_{q}^{\times} \\ n \neq 1}} \chi_{2}(n-1)=-\chi_{2}(-1)
$$

To evaluate the final sum, we multiply by $\chi_{2}^{2}\left(n^{-1}\right)$ to obtain

$$
\sum_{\substack{n \in F_{q}^{\times} \\ n \neq 1}} \chi_{2}(-n) \chi_{2}(n-1) \chi_{2}^{2}\left(n^{-1}\right)=\chi_{2}(-1) \sum_{\substack{n \in F_{q}^{\times} \\ n \neq 1}} \chi_{2}\left(1-n^{-1}\right)=-\chi_{2}(-1) .
$$

Hence,

$$
M\left(\chi_{2}\right)=\frac{1}{4}\left(q-2-3 \chi_{2}(-1)\right) .
$$

Applying Theorem 3.1 we deduce the following theorem:

THEOREM 4.1. If $\chi_{2}: \mathbb{F}_{q}^{\times} \longrightarrow \mathbb{C}^{\times}$is the quadratic character on $\mathbb{F}_{q}^{\times}$, then the digraph $D_{\chi_{2}}$ has

$$
T\left(D_{\chi_{2}}\right)=\frac{q(q-1)\left(q-2-3 \chi_{2}(-1)\right)}{24}
$$

directed triangles.

With the explicit enumeration of triangles in $D_{\chi_{2}}$ complete, we can consider the effect of lifting to a field extension of $\mathrm{F}_{q}$. Since the quadratic character case did not require us to appeal to the more general theory of Jacobi sums, it will not be necessary to use Davenport and Hasse's [3] work on character sums over finite field extensions. We will prove the following theorem.

THEOREM 4.2. Let $\chi_{2}: \mathbb{F}_{q}^{\times} \longrightarrow \mathbb{C}^{\times}$be the quadratic character on $\mathbb{F}_{q}^{\times}$and let $\psi_{2}=\chi_{2} \circ N_{r / q}: \mathbb{F}_{r}^{\times} \longrightarrow \mathbb{C}^{\times}$be the lifted character on $\mathbb{F}_{r}^{\times}=\mathbb{F}_{q^{s}}^{\times}$, where $s>1$. Then the number of directed triangles in the digraphs $D_{\chi_{2}}$ and $D_{\psi_{2}}$ 
are related by the identity

$$
3^{s-1}\left(q^{s}-2-\frac{24 T\left(D_{\psi_{2}}\right)}{q^{s}\left(q^{s}-1\right)}\right)=\left(q-2-\frac{24 T\left(D_{\chi_{2}}\right)}{q(q-1)}\right)^{s} .
$$

Proof. First, note that $N_{r / q}(-1)=(-1)^{s}$, from which we have $\psi_{2}(-1)=\chi_{2}(-1)^{s}$. From (1), it follows that

$$
M\left(\chi_{2}\right)=\frac{1}{4}\left(q-2-3 \chi_{2}(-1)\right) \quad \text { and } \quad M\left(\psi_{2}\right)=\frac{1}{4}\left(q^{s}-2-3 \chi_{2}(-1)^{s}\right)
$$

and we deduce the relation

$$
3^{s-1}\left(q^{s}-2-4 M\left(\psi_{2}\right)\right)=\left(q-2-4 M\left(\chi_{2}\right)\right)^{s} .
$$

Solving the main result of Theorem 3.1 for $M\left(\chi_{2}\right)$ (and $M\left(\psi_{2}\right)$ ) and plugging into the above relation yields the statement of the theorem.

\section{Cubic Characters}

In this section, we will compute $T\left(D_{\gamma_{3}}\right)$, where $\chi_{3}$ is a character of order 3 on $\mathbb{F}_{q}^{\times}$(known as a cubic character) and consider the effect of lifting to extension fields of $F_{q}$. It is assumed that the prime $p$ such that $q=p^{f}$ satisfies $p \equiv 1(\bmod 3)$, guaranteeing that such a character exists. When expanding $M\left(\chi_{3}\right)$, the cubic case yields nine sums, most of which are easily evaluated using the standard techniques that were implemented in the quadratic case. We have

$$
\begin{aligned}
& \sum_{\substack{n \in \mathrm{F}_{q}^{\times} \\
n \neq 1}} 1=q-2 \\
& \sum_{\substack{n \in \mathrm{F}_{q}^{\times} \\
n \neq 1}} \chi_{3}(-n)=\sum_{\substack{n \in \mathrm{F}_{q}^{\times} \\
n \neq 1}} \chi_{3}(n-1)=-\chi_{3}(-1)=-1 \\
& \sum_{\substack{n \in \mathrm{F}_{q}^{\times} \\
n \neq 1}} \chi_{3}^{2}(-n)=\sum_{\substack{n \in \mathrm{F}_{q}^{\times} \\
n \neq 1}} \chi_{3}^{2}(n-1)=-\chi_{3}^{2}(-1)=-1 \\
& \sum_{\substack{n \in \mathrm{F}_{q}^{\times} \\
n \neq 1}} \chi_{3}(-n) \chi_{3}^{2}(n-1)=\sum_{\substack{n \in \mathrm{F}_{q}^{\times} \\
n \neq 1}} \chi_{3}^{2}(-n) \chi_{3}(n-1)=-1
\end{aligned}
$$


The remaining sums

$$
\sum_{\substack{n \in \mathbb{F}_{q}^{\times} \\ n \neq 1}} \chi_{3}(-n) \chi_{3}(n-1) \quad \text { and } \quad \sum_{\substack{n \in \mathbb{F}_{q}^{\times} \\ n \neq 1}} \chi_{3}^{2}(-n) \chi_{3}^{2}(n-1)
$$

are conjugate Jacobi sums.

As our computation will require more sophisticated properties of Jacobi sums than were necessary in the quadratic case, we will need the solution on $\mathbb{F}_{p}^{\times}$in order to determine the value on $\mathbb{F}_{q}^{\times}$. Let $\phi_{3}: \mathbb{F}_{p}^{\times} \longrightarrow \mathbb{C}^{\times}$be either the cubic residue character or its conjugate. We will find that our answer is independent of this choice. Without loss of generality, we may assume that $\chi_{3}=\phi_{3} \circ N_{q / p}$.

From the assumption $p \equiv 1(\bmod 3)$, the prime $p$ splits in $Z[\omega]$ as $p=\pi \bar{\pi}$ (Proposition 9.1.4 of [6]). It will be necessary to pick a specific prime $\pi$ from among its six associates. The choice we make is that of a primary prime. In the cubic case, a primary prime $\pi=a+b \omega$ (where $\omega$ denotes the $3^{\text {rd }}$ root of unity $-\frac{1}{2}+\frac{\sqrt{3}}{2} i$ ) can be determined by the congruences

$$
a \equiv 2(\bmod 3) \quad \text { and } \quad b \equiv 0(\bmod 3)
$$

(see Section 9.3 of [6]).

In the case of the cubic residue character (or its conjugate) on $\phi_{3}: \mathrm{F}_{p}^{\times} \longrightarrow \mathbb{C}^{\times}$, the evaluations of the sums

$$
\sum_{\substack{n \in \mathbb{F}_{p}^{\times} \\ n \neq 1}} \phi_{3}(-n) \phi_{3}(n-1) \quad \text { and } \quad \sum_{\substack{n \in \mathbb{F}_{p}^{\times} \\ n \neq 1}} \phi_{3}^{2}(-n) \phi_{3}^{2}(n-1)
$$

are known and we find that their sum is equal to $2 a-b$, where $\pi=a+b \omega$ is a primary prime above $p$ in Z $[\omega]$ (see Lemma 1, Section 9.4 of [6]). Hence, we obtain the following theorem describing the number of triangles in $D_{\phi_{3}}$.

TheOREm 5.1. If $\phi_{3}: \mathbb{F}_{p}^{\times} \longrightarrow \mathbb{C}^{\times}$is a cubic character on $\mathbb{F}_{p}^{\times}$, then the digraph $D_{\phi_{3}}$ has

$$
T\left(D_{\phi_{3}}\right)=\frac{p(p-1)(p-8+2 a-b)}{81}
$$

directed triangles, where $\pi=a+b \omega$ is a primary prime above $p$ in $Z[\omega]$.

Now we return to $\chi_{3}: \mathbb{F}_{q}^{\times} \longrightarrow \mathrm{C}^{\times}$. In order to find the value of $T\left(D_{\chi_{3}}\right)$, it is necessary to appeal to Davenport and Hasse's [3] result on lifting Jacobi 
sums. In particular, we find that

$$
\begin{aligned}
\sum_{\substack{n \in \mathbb{F}_{q}^{\times} \\
n \neq 1}} \chi_{3}(-n) \chi_{3}(n-1)+\sum_{\substack{n \in F_{q}^{\times} \\
n \neq 1}} \chi_{3}^{2}(-n) \chi_{3}^{2}(n-1) & =-\left(J\left(\chi_{3}, \chi_{3}\right)+J\left(\chi_{3}^{2}, \chi_{3}^{2}\right)\right) \\
& =-\left(J\left(\phi_{3}, \phi_{3}\right)^{f}+J\left(\phi_{3}^{2}, \phi_{3}^{2}\right)^{f}\right) \\
& =-\left((-\pi)^{f}+(-\pi)^{f}\right) .
\end{aligned}
$$

Hence, we have

$$
T\left(D_{\chi_{3}}\right)=\frac{q(q-1)\left(q-8-\left((-\pi)^{f}+(\overline{-\pi})^{f}\right)\right)}{81},
$$

where $\pi$ is a primary prime above $p$ in $Z[\omega]$.

Now suppose that $\mathbb{F}_{r}$ is a finite extension of $\mathbb{F}_{q}$ satisfying $r=q^{s}$ and that $\psi_{3}:=\chi_{3} \circ N_{r / q}$. We will prove the following relationship between $T\left(D_{\chi_{3}}\right)$ and $T\left(D_{\psi_{3}}\right)$.

THEOREM 5.2. Let $\chi_{3}: \mathbb{F}_{q}^{\times} \longrightarrow \mathrm{C}^{\times}$be a cubic character on $\mathbb{F}_{q}^{\times}$(with $q=p^{f}$ and $\left.p \equiv 1(\bmod 3)\right)$ and let $\psi_{3}=\chi_{3} \circ N_{r / q}: \mathbb{F}_{r}^{\times} \longrightarrow \mathbb{C}^{\times}$be the lifted character on $\mathbb{F}_{r}^{\times}=\mathbb{F}_{q^{s}}^{\times}$, where $s>1$. Then the number of directed triangles in the digraphs $D_{\chi_{3}}$ and $D_{\psi_{3}}$ are related by the identity

$$
q^{s}-8-(-\pi)^{f_{s}}-\frac{81 T\left(D_{\psi_{3}}\right)}{q^{s}\left(q^{s}-1\right)}=\left(q-8-(-\pi)^{f}-\frac{81 T\left(D_{\chi_{3}}\right)}{q(q-1)}\right)^{s},
$$

where $\pi$ is a primary prime above $p$ in $Z[\omega]$.

Proof. If we let $\pi$ be a primary prime above $p$ in $Z[\omega]$, then we have shown that

$$
M\left(\chi_{3}\right)=\frac{1}{9}\left(q-8-(-\pi)^{f}-(-\pi)^{f}\right)
$$

and

$$
M\left(\psi_{3}\right)=\frac{1}{9}\left(q^{s}-8-(-\pi)^{f_{s}}-(-\pi)^{f_{s}}\right) .
$$

Solving the first equation for $(-\pi)^{f}$ and plugging into the second equation gives

$$
q^{s}-8-(-\pi)^{f_{s}}-9 M\left(\psi_{3}\right)=\left(q-8-(-\pi)^{f}-9 M\left(\chi_{3}\right)\right)^{s} .
$$

From Theorem 3.1, we are able to deduce the statement of the theorem. 


\section{Quartic Characters}

For the quartic case, assume that $p \equiv 1(\bmod 4)$ so that $p$ splits in Z[i] as $p=\varpi \bar{\varpi}$. One may choose from among the four associates of $\varpi$ by assuming that it is primary. This is equivalent to assuming that $\varpi=a+b i$ satisfies

$$
a \equiv 1(\bmod 4) \quad \text { and } \quad b \equiv 0(\bmod 4)
$$

or

$$
a \equiv 3(\bmod 4) \quad \text { and } \quad b \equiv 2(\bmod 4)
$$

(see Section 9.7 of [6]). As before, let $q=p^{f}$ and $\chi_{4}: \mathbb{F}_{q}^{\times} \longrightarrow \mathrm{C}^{\times}$be a quartic character.

Expanding $M\left(\chi_{4}\right)$ yields sixteen sums, ten of which are easily evaluated using the orthogonality relation:

$$
\begin{aligned}
& \sum_{\substack{n \in \mathrm{F}_{q}^{\times} \\
n \neq 1}} 1=q-2 \\
& \sum_{\substack{n \in \mathrm{F}_{q}^{\times} \\
n \neq 1}} \chi_{4}(-n)=\sum_{\substack{n \in \mathrm{F}_{q}^{\times} \\
n \neq 1}} \chi_{4}^{3}(-n)=-\chi_{4}(-1) \\
& \sum_{\substack{n \in \mathrm{F}_{q}^{\times} \\
n \neq 1}} \chi_{4}(n-1)=\sum_{\substack{n \in \mathrm{F}_{q}^{\times} \\
n \neq 1}} \chi_{4}^{3}(n-1)=-\chi_{4}(-1) \\
& \sum_{\substack{n \in \mathrm{F}_{q}^{\times} \\
n \neq 1}} \chi_{4}(-n) \chi_{4}^{3}(n-1)=\sum_{n \in \mathbb{F}_{q}^{\times}} \chi_{4}^{3}(-n) \chi_{4}(n-1)=-\chi_{4}(-1) \\
& \sum_{n \in \mathbb{F}_{q}^{\times}} \chi_{4}^{2}(-n)=\sum_{\substack{n \in \mathbb{F}_{q}^{\times} \\
n \neq 1}} \chi_{4}^{2}(n-1)=\sum_{\substack{n \in \mathbb{F}_{q}^{\times} \\
n \neq 1}} \chi_{4}^{2}(-n) \chi_{4}^{2}(n-1)=-1 .
\end{aligned}
$$

The remaining six sums are given by

$$
S(i, j)=\sum_{\substack{n \in \mathbb{F}_{q}^{\times} \\ n \neq 1}} \chi_{4}^{i}(-n) \chi_{4}^{j}(n-1),
$$

where $i, j=1,2,3$ and $i+j \neq 4$. We may evaluate them using standard techniques of Jacobi sums. It is easily checked that $S(1,1)=S(1,2)=$ $S(2,1)$ and $S(3,3)=S(3,2)=S(2,3)$, implying that

$$
M\left(\chi_{4}\right)=\frac{1}{16}\left(q-5-6 \chi_{4}(-1)-3\left(J\left(\chi_{4}, \chi_{4}\right)+\overline{J\left(\chi_{4}, \chi_{4}\right)}\right) .\right.
$$


Following our approach in the cubic case, let $\phi_{4}: \mathbb{F}_{p}^{\times} \longrightarrow \mathbb{C}^{\times}$be either the quartic residue character or its conjugate. Without loss of generality, assume that $\chi_{4}=\phi_{4} \circ N_{q / p}$. It is known that

$$
J\left(\phi_{4}, \phi_{4}\right)+\overline{J\left(\phi_{4}, \phi_{4}\right)}=\phi_{4}(-1)(\varpi+\bar{\varpi})
$$

(see Proposition 9.9.4 of [6]), implying the following theorem.

THEOREM 6.1. If $\phi_{4}: \mathbb{F}_{p}^{\times} \longrightarrow \mathbb{C}^{\times}$is a quartic character on $\mathbb{F}_{p}^{\times}$, then the digraph $D_{\phi_{4}}$ has

$$
T\left(D_{\phi_{4}}\right)=\frac{p(p-1)\left(p-5-6(-1)^{(p-1) / 4}(a+1)\right)}{192}
$$

directed triangles, where $\varpi=a+b i$ is a primary prime above $p$ in Z[i].

Applying Davenport and Hasse's result [3], we deduce that

$$
T\left(D_{\chi_{4}}\right)=\frac{q(q-1)\left(q-5-6 \chi_{4}(-1)+\left((-1)^{(p-1) / 4} \varpi\right)^{f}+\left((-1)^{(p-1) / 4} \varpi\right)^{f}\right)}{192} .
$$

Finally, assume that $r=q^{s}$ with $s>1$ and $\psi_{4}: \mathbb{F}_{r}^{\times} \longrightarrow \mathbb{C}^{\times}$is given by $\psi_{4}=\chi_{4} \circ N_{r / q}$. We will describe the relationship between $T\left(D_{\chi_{4}}\right)$ and $T\left(D_{\psi_{4}}\right)$. The details of the proof are left to the reader as they are essentially the same as was needed to prove Theorem 5.2.

TheORem 6.2. Let $\chi_{4}: \mathbb{F}_{q}^{\times} \longrightarrow \mathbb{C}^{\times}$be a quartic character on $\mathbb{F}_{q}^{\times}$(with $q=p^{f}$ and $\left.p \equiv 1(\bmod 4)\right)$ and let $\psi_{4}=\chi_{4} \circ N_{r / q}: \mathbb{F}_{r}^{\times} \longrightarrow \mathbb{C}^{\times}$be the lifted character on $\mathbb{F}_{r}^{\times}=\mathbb{F}_{q^{s}}^{\times}$, where $s>1$. Then the number of directed triangles in the digraphs $D_{\chi_{4}}$ and $D_{\psi_{4}}$ are related by the identity

$$
\begin{aligned}
& 3^{s-1}\left(q^{s}-5-6 \chi_{4}(-1)^{s}-\frac{192 T\left(D_{\psi_{4}}\right)}{q^{s}\left(q^{s}-1\right)}-3 \varpi^{f s}\right) \\
& =\left(q-5-6 \chi_{4}(-1)-\frac{192 T\left(D_{\chi_{4}}\right)}{q(q-1)}-3 \varpi^{f}\right)^{s},
\end{aligned}
$$

where $\varpi$ is a primary prime above $p$ in Z[i].

\section{Conclusion}

While the methods applied for counting triangles in the quadratic, cubic, and quartic character difference digraphs could be generalized, the 
sheer number of sums considered for higher-ordered characters imposes some limitations to this approach. Furthermore, the difficulty in evaluating Jacobi sums make this task even more intimidating. However, Berndt, Evans, and Williams provide us in [1] with the necessary background to perform calculations in certain higher-ordered cases. Future endeavors involving such calculations could help strengthen the tie that binds number theory and graph theory while providing us with a deeper understanding for these character difference digraphs.

\section{REFERENCES}

[1] B. Berndt - R. Evans - K. Williams, Gauss and Jacobi Sums, Canad. Math. Soc. Series of Monographs and Advanced Texts, 21, John Wiley \& Sons, Inc., 1998.

[2] M. Budden - N. Calkins - W. Hack - J. Lambert - K. Thompson, Enumeration of Triangles in Quartic Residue Graphs, INTEGERS, 11 (2011), \# A48.

[3] H. Davenport - H. Hasse, Die Nullstellen der Kongruenzzetafunktionen in gewissen zyklischen Fällen, J. Reine Angew. Math., 172 (1934), pp. 151-182.

[4] P. Erdos - A. RÉnYi, Asymmetric Graphs, Acta Math. Acad. Sci. Hun., 14 (1963), pp. 295-315.

[5] R. Graham - J. Spencer, A Constructive Solution to a Tournament Problem, Canad. Math. Bull., 14 (1971), pp. 45-48.

[6] K. Ireland - M. Rosen, A Classical Introduction to Modern Number Theory, $2^{\text {nd }}$ edition, Springer-Verlag, 1990.

[7] J. Kendall - B. Smith, On the method of paired comparisons, Biometrika, 31 (1940), pp. 324-345.

[8] F. Lemmermeyer, Reciprocity Laws, Springer Monographs in Mathematics, Springer-Verlag, 2000.

[9] T. Lim - C. Praeger, On generalised Paley graphs and their automorphism groups, Michigan Math. J., 58 (2009), pp. 293-308.

[10] B. Maheswari - M. LAVAKU, Enumeration of Triangles and Hamilton Cycles in Quadratic Residue Cayley Graphs, Chamchuri J. Math., 1 (2009), pp. 95-103.

[11] R. E. A. C. Paley, On Orthogonal Matrices, J. Math. Phys., 12 (1933), pp. 311-320.

[12] H. SACHS, Über Selbstkomplementäre Graphen, Publ. Math. Debrecen, 9 (1962), pp. 270-288.

Manoscritto pervenuto in redazione il 27 agosto 2013. 
